\title{
Pediatric sarcomas: translating molecular pathogenesis of disease to novel therapeutic possibilities
}

\author{
Jennifer L. Anderson', Christopher T. Denny ${ }^{2,3}$, William D. Tap ${ }^{4}$ and Noah Federman ${ }^{3}$
}

Pediatric sarcomas represent a diverse group of rare bone and soft tissue malignancies. Although the molecular mechanisms that propel the development of these cancers are not well understood, identification of tumor-specific translocations in many sarcomas has provided significant insight into their tumorigenesis. Each fusion protein resulting from these chromosomal translocations is thought to act as a driving force in the tumor, either as an aberrant transcription factor (TF), constitutively active growth factor, or ligand-independent receptor tyrosine kinase. Identification of transcriptional targets or signaling pathways modulated by these oncogenic fusions has led to the discovery of potential therapeutic targets. Some of these targets have shown considerable promise in preclinical models and are currently being tested in clinical trials. This review summarizes the molecular pathology of a subset of pediatric sarcomas with tumor-associated translocations and how increased understanding at the molecular level is being translated to novel therapeutic advances.

S arcomas are a rare, heterogeneous group of neoplasms presumed to be of mesenchymal origin. Tumors can arise in bone or soft tissues such as muscle and fat and can develop anywhere in the body. They account for only $1 \%$ of all malignancies, and although the incidence of malignancy is higher in adults, sarcomas occur with higher frequency in children. Each year, between 1,500 and 1,600 children and young adults in the United States develop these malignant bone and soft tissue tumors comprising $\sim 13 \%$ of cancers afflicting patients below the age of $20(1,2)$. The overall 5-year survival rate for pediatric sarcomas is $\sim 60 \%$ and falls closer to $20-30 \%$ for recurrent and metastatic cases despite aggressive surgery, multiagent chemotherapy, and/or radiation.

Sarcomas can be divided into two groups based on the underlying molecular events that initiate tumorigenesis. The first group is characterized by the presence of specific chromosomal translocations (Table 1) or activating mutations whereas the second is more cytogenically complex. Sarcomas with complex karyotypes predominantly afflict older patients and translocations tend to be observed with higher frequency in pediatric cases. Pediatric sarcomas with tumor-associated translocations include Ewing sarcoma (ES), rhabdomyosarcoma (RMS), synovial sarcoma (SS), and dermatofibrosarcoma protuberans (DFSP). The genetic aberrations in these neoplasms produce defined fusions that are critical for sarcomagenesis. Depending on the genes involved in the fusion, the resulting protein can promote tumor progression through transcriptional modulation, epigenetic modifications, or activation of oncogenic signaling pathways.

Due to the rarity of sarcomas, especially when considering the prevalence of individual subtypes, they are understudied cancers. As the fusion proteins present within translocationassociated sarcomas are inherent to tumor development, they provide an avenue of research for development of improved and targeted treatments. This review focuses on four pediatric sarcomas with tumor-associated translocations and will discuss the molecular genetics of these malignancies, potential therapeutic targets, and the status of agents directed against these targets in clinical trials.

\section{ES}

ES is part of a group of bone and soft tissue small-round blue cell malignancies that predominantly occur within the second decade of life. ES is the second most common bone malignancy in children and adolescents, with an annual incidence of 2.93 per million in the United States (3); it accounts for 3\% of pediatric cancers. The 5 -year survival rate is nearing $70 \%$, although this number shrinks to $\sim 20-30 \%$ in patients with metastatic, refractory, and/or recurrent disease (4). The current treatment of ES is multimodal consisting of intensive multiagent chemotherapy, surgery, and/or high-dose radiation therapy. The standard systemic chemotherapeutic regimen includes cycles of vincristine, cyclophosphamide, and doxorubicin with ifosfamide and etoposide.

ES occurs primarily as tumors of the bone, with less than $15 \%$ of cases arising in extraosseous locations (5). ES primary osseous sites are split between the extremities and the central axis, with an increased tendency for incidence in the shaft of long tubular bones, pelvis, and rib. The histogenesis of ES has long been disputed, as evidence has been provided for both a neural crest and mesenchymal stem cell origin. 
Table 1. Sarcomas with defined chromosomal translocations

\begin{tabular}{|c|c|c|}
\hline Tumor type & Translocation & Fusion \\
\hline \multirow[t]{9}{*}{ ES } & $\mathrm{t}(11 ; 22)(\mathrm{q} 24 ; \mathrm{q} 12)$ & EWS-FLI1 \\
\hline & $t(21 ; 22)(q 22 ; q 12)$ & EWS-ERG \\
\hline & $t(7 ; 22)(p 22 ; q 12)$ & EWS-ETV1 \\
\hline & $\mathrm{t}(17 ; 22)(\mathrm{q} 21 ; \mathrm{q} 12)$ & EWS-ETV4 \\
\hline & $\mathrm{t}(2 ; 22)(\mathrm{q} 33 ; \mathrm{q} 12)$ & EWS-FEV \\
\hline & $t(16 ; 21)(p 11 ; q 22)$ & FUS-ERG \\
\hline & $\mathrm{t}(2 ; 16)(q 35 ; p 11)$ & FUS-FEV \\
\hline & $(t(1 ; 22)(p 36.1 ; q 12)$ & EWS-ZSG \\
\hline & $\mathrm{t}(20 ; 22)(q 13 ; q 12)$ & EWS-NFATC2 \\
\hline \multirow{4}{*}{$\begin{array}{l}\text { ES-like tumors } \\
\text { (CD-99 negative) }\end{array}$} & $t(6 ; 22)(p 21 ; q 12)$ & EWS-POU5F1 \\
\hline & $\mathrm{t}(1 ; 22)(\mathrm{q} 36.1 ; \mathrm{q} 12)$ & EWS-PATZI \\
\hline & $\mathrm{t}(2 ; 22)(\mathrm{q} 31 ; \mathrm{q} 12)$ & EWS-SP3 \\
\hline & $\mathrm{t}(4 ; 19)(\mathrm{q} 35 ; \mathrm{q} 13)$ & $C I C-D U X 4$ \\
\hline \multirow[t]{2}{*}{ Clear-cell sarcoma } & $t(12 ; 22)(p 13 ; q 12)$ & EWS-ATF1 \\
\hline & $\mathrm{t}(2 ; 22)(\mathrm{q} 33 ; \mathrm{q} 12)$ & EWS-BREB 1 \\
\hline \multirow{2}{*}{$\begin{array}{l}\text { Desmoplastic } \\
\text { small-round-cell tumor }\end{array}$} & $t(11 ; 22)(p 13 ; q 12)$ & EWS-WT1 \\
\hline & $t(21 ; 22)(q 22 ; q 12)$ & EWS-ERG \\
\hline \multirow[t]{2}{*}{ Myxoid liposarcoma } & $t(12 ; 16)(q 13 ; q 11)$ & FUS-DDIT3 \\
\hline & $\mathrm{t}(12 ; 22)(q 13 ; q 12)$ & EWS-DDIT3 \\
\hline \multirow{4}{*}{$\begin{array}{l}\text { Extraskeletal myxoid } \\
\text { chondrosarcoma }\end{array}$} & $\mathrm{t}(9 ; 22)(\mathrm{q} 22-31 ; \mathrm{q} 11-12)$ & EWS-NR4A3 \\
\hline & $\mathrm{t}(9 ; 17)(\mathrm{q} 22 ; \mathrm{q} 11)$ & TAF15-NR4A3 \\
\hline & $\mathrm{t}(9 ; 15)(\mathrm{q} 22 ; \mathrm{q} 21)$ & TCFI2-NR4A3 \\
\hline & $\mathrm{t}(9 ; 22)(\mathrm{q} 22 ; \mathrm{q} 15)$ & TFG-NR4A3 \\
\hline \multirow{2}{*}{$\begin{array}{l}\text { Low-grade fibromyxoid } \\
\text { sarcoma }\end{array}$} & $\mathrm{t}(7 ; 16)(q 33 ; p 11)$ & FUS-CREB3L2 \\
\hline & $t(11 ; 16)(p 11 ; p 11)$ & FUS-CREB3L1 \\
\hline \multirow{3}{*}{$\begin{array}{l}\text { Angiomatoid fibrous } \\
\text { histiocytoma }\end{array}$} & $t(12 ; 16)(q 13 ; p 11)$ & FUS-ATF1 \\
\hline & $\mathrm{t}(12 ; 22)(q 13 ; q 12)$ & EWS-ATF1 \\
\hline & $\mathrm{t}(2 ; 22)(\mathrm{q} 33 ; \mathrm{q} 12)$ & EWS-CREB 1 \\
\hline \multirow{5}{*}{$\begin{array}{l}\text { Alveolar } \\
\text { rhabdomyosarcoma }\end{array}$} & $\mathrm{t}(2 ; 13)(\mathrm{q} 35 ; \mathrm{q} 14)$ & PAX3-FOXO1 \\
\hline & $t(1 ; 13)(q 36 ; q 14)$ & PAX7-FOXO1 \\
\hline & $t(2 ; 2)(p 23 ; q 35)$ & PAX3-NCOA 1 \\
\hline & $\mathrm{t}(2 ; 8)(\mathrm{q} 35 ; \mathrm{q} 13)$ & PAX3-NCOA2 \\
\hline & $\mathrm{t}(8 ; 13 ; 9)(\mathrm{p} 11.2 ; q 14 ; 9 q 32)$ & FGFR1-FOXO1 \\
\hline $\begin{array}{l}\text { Alveolar soft part } \\
\text { sarcoma }\end{array}$ & $t(X ; 17)(p 11 ; q 25)$ & ASPSL-TFE3 \\
\hline $\begin{array}{l}\text { Congenital } \\
\text { fibrosarcoma }\end{array}$ & $t(12 ; 15)(p 13 ; q 25)$ & ETV6-NTRK3 \\
\hline $\begin{array}{l}\text { Congenital mesoblastic } \\
\text { nephroma }\end{array}$ & $t(12 ; 15)(p 13 ; q 25)$ & ETV6-NTRK3 \\
\hline \multirow{4}{*}{$\begin{array}{l}\text { Inflammatory } \\
\text { myofibroblastic tumor }\end{array}$} & $\mathrm{t}(1 ; 2)(q 25 ; q 23)$ & TPM3-ALK \\
\hline & $\mathrm{t}(2 ; 19)(\mathrm{q} 23 ; \mathrm{q} 13)$ & TPM4-ALK \\
\hline & $\mathrm{t}(2 ; 17)(\mathrm{q} 23 ; \mathrm{q} 23)$ & CLTC-ALK \\
\hline & $\mathrm{t}(2 ; 2)(p 23 ; q 13)$ & RANBP2-ALK \\
\hline \multirow[t]{4}{*}{ Synovial sarcoma } & $t(X ; 18)(p 11 ; q 11)$ & SS18-SSX1 \\
\hline & $t(X ; 18)(p 11 ; q 11)$ & SS18-SSX2 \\
\hline & $\mathrm{t}(\mathrm{X} ; 18)(\mathrm{p} 11 ; \mathrm{q} 13)$ & SS18-SSX4 \\
\hline & $t(X ; 20)(p 11 ; q 13)$ & SS18L1-SSX1 \\
\hline \multirow[t]{3}{*}{ Endometrial sarcoma } & $\mathrm{t}(7 ; 17)(\mathrm{p} 15 ; \mathrm{q} 21)$ & JAZF1-SUZ12 \\
\hline & $\mathrm{t}(6 ; 7)(\mathrm{p} 21 ; \mathrm{p} 15)$ & JAZF1-PHF1 \\
\hline & $\mathrm{t}(6 ; 10)(\mathrm{p} 21 ; \mathrm{p} 11)$ & EPC1-PHF1 \\
\hline $\begin{array}{l}\text { Dermatofibrosarcoma } \\
\text { protuberans }\end{array}$ & $t(17 ; 22)(q 22 ; q 13)$ & COL1A1-PDGFB \\
\hline Giant cell fibroblastoma & $\mathrm{t}(17 ; 22)(\mathrm{q} 22 ; \mathrm{q} 13)$ & COL1A1-PDGFB \\
\hline
\end{tabular}

ES, Ewing sarcoma.

\section{Molecular genetics}

The pathognomonic genetic aberration in ES fuses the EWS gene (also known as EWSR1, ES break point region 1) to one of five ETS (erythroblast transformation-specific) transcription factors (TFs) (Table 1). FLI1 (Friend leukemia virus integration 1 ) is the fusion partner in $\sim 85 \%$ of cases and $E R G$ (v-ets erythroblastosis virus E26 oncogene homolog) in about 10\%, whereas others each account for less than $1 \%$ (6). In very rare cases, FUS combines with ERG or FEV (ETS oncogene family), and EWS is juxtaposed to non-ETS genes (7) (Table 1). Adding to the complexity of multiple translocations, variation in the location of the chromosomal break point results in numerous types of each fusion. Initial reports suggested EWS-FLI1 type 1 fusions confer a prognostic advantage to patients with localized disease, but more recent studies have demonstrated there is no difference in clinical outcome based on fusion type (8).

The most common EWS-ETS fusion, EWS-FLI1, is generated from the $t(11 ; 22)(\mathrm{q} 24 ; \mathrm{q} 12)$ reciprocal translocation that combines the $\mathrm{N}$-terminal EWS activation domain and the C-terminal ETS DNA-binding domain of FLI1 (9) (Figure 1a). EWS is a member of the FET (FUS, EWS, and TAF15) family of proteins, which may be involved in transcription and mRNA splicing as they contain both an activation and RNAbinding domain (10). The ETS family constitutes a group of 30 TFs characterized by the presence of a highly conserved ETS domain that mediates site-specific DNA binding. They also possess either an activation or a repression domain and are involved in various cellular processes such as cell proliferation and differentiation (11). Because the more potent EWS activation domain replaces that of FLI1 while the FLI1 DNA-binding domain remains intact, EWS-FLI1 is thought to primarily act as an aberrant TF. As most ETS TFs bind to a core consensus (GGAA/T), EWS/FLI1 has also been shown to interact with DNA in a site-specific manner through association with GGAA microsatellites (12). Interactions with splicing factors and the ability to alter splice-site selection suggest EWS-FLI1 also plays a role in RNA splicing $(13,14)$. The capacity of EWS-FLI1 to function post-transcriptionally is further demonstrated by modulation of target gene RNA half-life (15) (Figure 1b).

\section{Target genes and targeted therapies}

Ectopic expression of EWS-FLI1 in heterologous cell types or small interfering RNA-mediated knockdown of the fusion in ES cell lines have both been used to discover potential target genes. These studies have identified both up- and downregulated genes, demonstrating the function of EWS-FLI1 as both a transcriptional activator and a repressor. Multiple direct targets have been confirmed through demonstration of EWSFLI1 binding to their promoters including insulin-like growth factor (IGF) binding protein 3 (16) and the Aurora $A$ and $B$ kinases (17).

Repression of IGF binding protein 3 by EWS-FLI1 is one of the multiple connections between the IGF pathway and ES pathogenesis. In addition to targeting IGF binding protein 3, EWS-FLI1 has been shown to upregulate IGF1 in mesenchymal progenitor cells (18). In addition, ES cell lines ubiquitously 

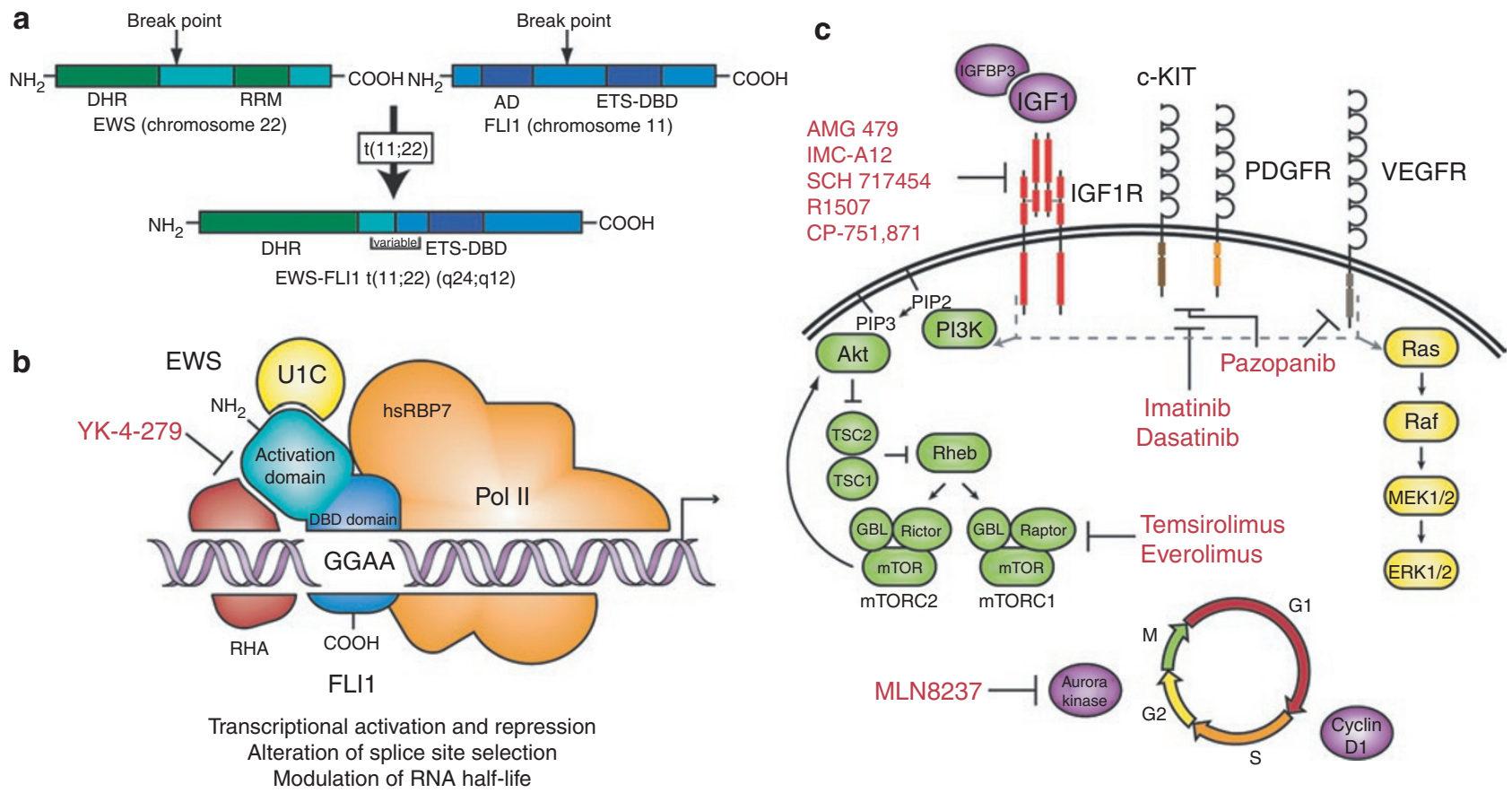

Figure 1. Molecular genetics and targeted therapies in ES. (a) Schematic of EWS-FLI1 t(11;22)(q24;q12) translocation. The EWS-FLI1 fusion includes the N-terminal activation domain of EWS, which contains multiple degenerate hexapeptide repeats (consensus SYGQQS), and the C-terminal ETS DNA-binding domain (ETS-DBD) of FLI1. The RNA recognition motif (RRM) of EWS and the activation domain (AD) of FLI1 are not retained in the fusion. Variation in the sites of chromosomal break points leads to multiple fusion types (bracketed region). (b) Putative molecular function of the EWS-FLI1 protein and selected protein-protein interactions. As an aberrant transcription factor, EWS-FLI1 regulates genes in part by binding to GGAA microsatellites upstream of target genes. EWS-FLI1 has been shown to interact with the splicing factor U1C (also known as SNRPC, small nuclear ribonucleoprotein polypeptide c), RNA helicase A (RHA), and the hRBP7 subunit of RNA polymerase II (Pol II), which links the protein to splicing and transcription. The small molecule that blocks the EWS-FLI1-RHA interaction is indicated in red. (c) Signaling pathways and targeted therapies in ES. EWS-FLI1 modulation of IGFBP3 and IGF1 and overexpression of IGF1R promote increased IGF1 signaling. ES also expresses PDGFR, c-KIT, and VEGFR. Activation of IGF1R, PDGFR, c-KIT, and VEGFR leads to downstream signaling through the PI3K and MAPK pathways (indicated by gray dashed line and arrows). EWS-FLI1 upregulates Aurora kinase A and cyclin D1, promoting progression through the cell cycle. Targeted therapeutic agents used in recent clinical trials for ES are indicated in red. Genes modulated by EWS-FLI1 are indicated in purple. Receptors overexpressed in ES are indicated in red. ES, Ewing sarcoma; ETS, erythroblast transformation-specific; FLI1, Friend leukemia virus integration 1; IGFBP, insulin-like growth factor binding protein; IGF1, insulin-like growth factor 1; IGF1R, IGF1 receptor; MAPK, mitogen-activated protein kinase; PDGF, platelet-derived growth factor; PDGFR, PDGF receptor; PI3K, phosphoinositide-3kinase; VEGFR, vascular endothelial growth factor receptor.

express the IGF1 receptor (IGF1R) and display autocrine production of IGF1. Moreover, IGF1R is necessary for EWS/FLI1mediated cellular transformation and inhibition of IGF1R suppresses tumor growth in vitro and in vivo (19). In the clinic, phase I trials of monoclonal antibodies targeting IGF1R (Figure 1c) have shown partial and complete responses in patients with ES (20). Although these early results were promising, recent phase II studies showed limited response rates of $\sim 10 \%$ for patients with recurrent or refractory ES (21-23). To improve the efficacy of anti-IGF1R therapies, future work is being directed toward identification of predictive biomarkers associated with patients with ES who benefit from treatment or combination therapy with other targeted agents (Table 2).

Although the IGF pathway has received the most attention, other EWS-FLI1 target genes or interacting proteins provide potential therapeutic targets. EWS-FLI1 upregulates both Aurora A and Aurora B, cell cycle-regulated serine/threonine kinases that are overexpressed in multiple cancers (17). Preclinical testing revealed a maintained complete clinical response for an Aurora kinase A inhibitor (Figure 1c) in an ES xenograft model (24). A phase II trial evaluating the effects of this drug in pediatric leukemias and solid tumors, including ES, is currently under way (NCT01154816, Table 2). It has also been shown that RNA helicase A, a protein involved in the regulation of transcription and splicing, binds to EWS-FLI1 and enhances its transcriptional activity (25). Utilization of a small molecule inhibitor to block the RNA helicase A-EWS/FLI1 interaction (Figure 1b) induces apoptosis in ES cells and reduces tumor growth in xenografts (26). This promising preclinical evidence suggests these agents may be effective in clinical trials.

\section{ALVEOLAR RMS (ARMS)}

RMS is the most common pediatric soft tissue sarcoma, accounting for $5-7 \%$ of all malignancies in children and adolescents less than 20 years of age. The overall 5-year survival rate is $\sim 60 \%$, although it is only $30 \%$ for those with metastatic disease (27). Current therapy for RMS is similar to that for ES, employing a combination of adjuvant intensive chemotherapy with surgery and/or radiation to the primary and metastatic sites of disease. 
Table 2. Targeted agents undergoing clinical testing for pediatric sarcomas

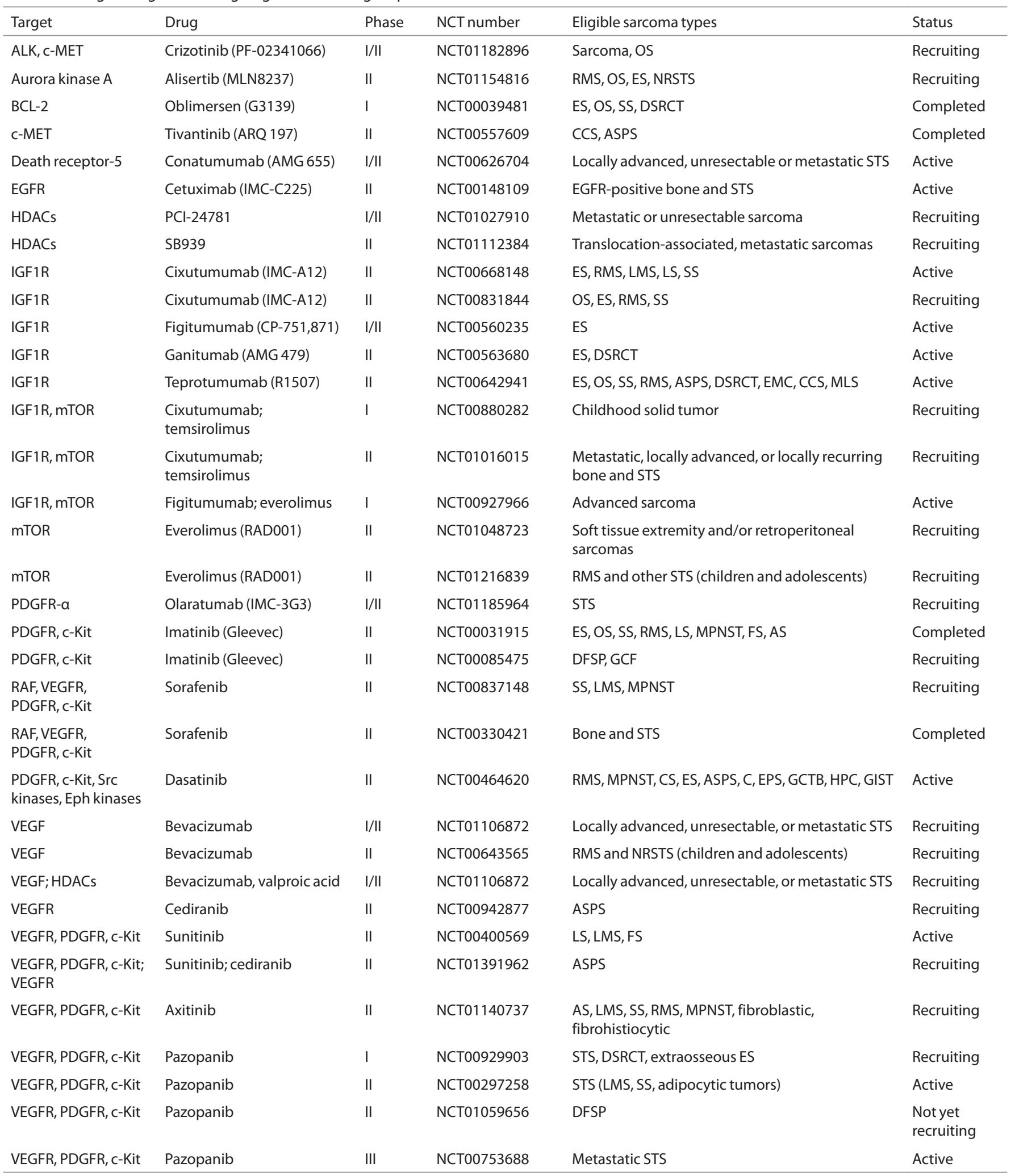

AS, angiosarcoma; ALK, anaplastic lymphoma receptor tyrosine kinase; ASPS, alveolar soft part sarcoma; BCL-2, B-cell CLL/lymphoma 2; C, chordoma; CCS, clear-cell sarcoma; CS, chondrosarcoma; C-MET: met proto-oncogene (hepatocyte growth factor receptor); c-KIT, v-kit Hardy-Zuckerman 4 feline sarcoma viral oncogene homolog; DFSP, dermatofibrosarcoma protuberans; DSRCT, desmoplastic small-round-cell tumor; EGFR, epidermal growth factor receptor; EMC, extraskeletal myxoid chondrosarcoma; EPS, epitheliod sarcoma; ES, Ewing sarcoma; FS, fibrosarcoma; GCF, giant cell fibrosarcoma; GCTB, giant cell tumor of bone; GIST, gastrointenstinal stromal tumor; HDACs, histone deacetylases; HPC, hemangiopericytoma; IGF1R, insulin-like growth factor 1 receptor; LMS, leiomyosarcoma; LS, liposarcoma; MLS, myxoid liposarcoma; MPNST, malignant peripheral nerve sheath tumor; mTOR, mammalian target of rapamycin; NRSTS, nonrhabdomyosarcoma soft tissue sarcoma; OS, osteosarcoma; PDGF, platelet-derived growth factor; RAF, v-raf-1 murine leukemia viral oncogene homolog 1; RMS, rhabdomyosarcoma; SS, synovial sarcoma; Src, v-src sarcoma (Schmidt-Ruppin A-2) viral oncogene homolog (avian); STS, soft tissue sarcoma; VEGFR, vascular endothelial growth factor receptor. 


\section{Review $\mid$ Anderson e tal.}

RMS is divided into histologic subtypes. Embryonal RMS (ERMS) and ARMS are the two major subtypes, accounting for $\sim 60 \%$ and $\sim 20 \%$ of cases, respectively (2). RMS tumors can arise anywhere in the body, although frequency of primary sites varies with histological subtype and age of diagnosis. Tumors in unfavorable sites (e.g., extremities) are more common in ARMS, which predominantly afflicts adolescents and young adults and confers a worse prognosis than ERMS (28). For patients who have refractory, recurrent, and/or metastatic ARMS, long-term survival is truly the exception rather than the rule. Although the expression of skeletal muscle markers and location of tumors in skeletal muscle suggest a myogenic origin, the exact cell of origin for ARMS remains uncertain. Mouse models and in vitro cell culture systems have provided evidence for both skeletal muscle and mesenchymal stem cell origins.

\section{Molecular genetics}

In addition to histologic differences, ARMS is distinguished from ERMS by the presence of specific chromosomal translocations present in the majority of ARMS tumors. The predominant translocation, $\mathrm{t}(2 ; 13)(\mathrm{q} 35 ; \mathrm{q} 14)$, fuses $P A X 3$ (paired box 3 ) to forkhead in human rhabdomyosarcoma (FOXO1) (forkhead box O1, also known as FKHR) (29). Less commonly, the $\mathrm{t}(1 ; 13)(\mathrm{p} 36 ; \mathrm{q} 14)$ translocation fuses another $P A X$ gene, $P A X 7$ (paired box 7), to FOXO1 (30). A recent study also identified rare, noncanonical $\mathrm{t}(2 ; 2)(\mathrm{p} 23 ; 35)$, and $\mathrm{t}(2: 8)(\mathrm{q} 35 ; \mathrm{q} 13)$ translocations that unite $P A X 3$ with the nuclear receptor transcriptional coactivators NCOA1 or NCOA2 (31) (Table 1).

PAX3 and PAX7 are part of the paired box family of TFs, which is involved in embryonic development and myogenesis (32). FOXO1 is a member of a subfamily of forkhead TFs regulated by the PI3K (phosphoinositide-3-kinase) pathway and is believed to play a role in myogenic growth and differentiation (33). Both translocation break points consistently occur within the seventh intron of the $P A X$ gene and the first intron of FOXO1, resulting in a chimeric TF that contains the PAX DNA-binding domain and transcriptional activation domain of FOXO1. Fusion type has been found to correlate with clinical outcome, as patients with PAX7-FOXO1-positive tumors had better overall survival rates than those with tumors containing the PAX3-FOXO1 fusion (34). More recently, clinical characteristics and prognosis of fusion-negative ARMS were found to be more similar to ERMS than fusion-positive ARMS, implying that the presence of the $P A X-F O X O 1$ fusion is more crucial than histology to the underlying biology of the tumor (35).

\section{Target genes and targeted therapies}

Initial PAX3-FOXO1 target genes were identified by evaluating the expression of known PAX3 target genes in ERMS cells transduced with PAX3-FOXO1. Upregulation of MET (met proto-oncogene/hepatocyte growth factor receptor) upon PAX3-FOXO1 expression and an observed correlation between MET and PAX3-FOXO1 in tumor samples suggested MET is a downstream target (36). The role of MET in ARMS tumorigenesis was further characterized by experiments that demonstrated MET is required for PAX3-FOXO1-mediated transformation of mouse embryonal fibroblasts, and short hairpin RNA knockdown of MET results in decreased tumor growth in vitro and in vivo (37). More recently, studies have shown that MET is posttranscriptionally regulated, as low levels of the microRNAs miR-1 and miR-206 result in derepression and upregulation of MET in RMS cells. Furthermore, overexpression of these miRNAs promoted myogenic differentiation and inhibited tumor growth in vivo (38). Additionally, a preclinical study demonstrated the ability of a c-MET small molecule inhibitor to hinder growth of ARMS cell lines (39).

Components of the IGF system are also potential therapeutic targets in ARMS. Heterologous expression of the PAX3FOXO1 fusion results in an increase in IGF1R levels and overexpression of IGF2 and IGF1R has been observed in ARMS and ERMS tumors and cell lines $(40,41)$. Elevated IGF2 levels in ERMS result from loss of heterozygosity at the 11p15.5 locus whereas in ARMS it may be transcriptionally upregulated. Introduction of PAX3-FOXO1 into NIH3T3 cells identified a myogenic transcriptional signature distinct from PAX3 alone that included genes such as those encoding MyoD, myogenin, and IGF2 (42). Multiple studies have demonstrated suppression of tumor growth in vivo and in vitro with small molecular inhibitors and monoclonal antibodies targeting IGF1R. This has led to both phase I and II clinical trials to evaluate antiIGF1R therapy in RMS. In phase I trials involving multiple tumor types, responses were observed only in patients with ES. However, preliminary phase II data have shown three objective radiological responses for patients with RMS (41).

The platelet-derived growth factor a receptor (PDGFR $\alpha$ ) is also transcriptionally upregulated by PAX3-FOXO1 (43). PDGFRa is overexpressed in human ARMS and ERMS tumors as well as in mouse models of ARMS. Small interfering RNA downregulation of PDGFRa resulted in decreased cell growth in vitro and PDGFRa inhibition in mouse models using imatinib mesylate (Gleevec) or a PDGFRa neutralizing antibody led to disease stabilization and in some cases resistance to therapy (44). Resistance has also been observed with anti-IGF1R therapy, which may be due to activation of other growth factor receptors such as human epidermal growth factor receptor 2 (also known as ERBB2) and PDGFRa $(45,46)$. Because both IGF1R and PDGFRa are potential therapeutic targets that have shown resistance as single agents, combination therapy may enhance patient response. Combination of anti-IGF1R therapy with mTOR (mammalian target of rapamycin) inhibitors is currently being evaluated in a phase I trial for pediatric solid tumors (NCT00880282) and in combination with cytotoxic chemotherapy in a Children's Oncology Group study (ARST08P1) for patients with high-risk RMS (Table 2).

\section{SS}

SS is the most common non-RMS soft tissue sarcoma in adolescents and young adults, accounting for $\sim 8 \%$ of all soft tissue sarcomas (28). The overall 5-year survival rate is between 70 and $80 \%$, but as with most sarcomas, this number drops sharply for those patients with metastatic and recurrent disease. Other 
than complete surgical resection of localized SSs, there is no standard of care for treatment and approach to these tumors may vary from center to center. Radiation therapy may be used as an adjuvant therapy to improve local control, and chemotherapy is generally reserved for larger tumors or patients with metastatic disease.

SS is histologically unique, displaying both biphasic and monophasic tumors. Monophasic tumors are mesenchymal in origin, exhibiting a spindle cell morphology that is difficult to distinguish from fibrosarcoma. Biphasic tumors contain cells of epithelial differentiation that form a glandular component within the mesenchymal spindle cells (28). The designation of SS originated from tumor proximity to large joints, as most tumors arise in the extremities, and microscopic resemblance to synovial tissue. However, this is a misnomer as the histogenesis is not of synovial origin (46). Mouse models utilizing myogenic regulatory factor-driven expression of fusion genes suggest SS arises shortly after differentiation from a muscle stem cell (47).

\section{Molecular genetics}

The underlying genetic aberration in SS results from a specific $\mathrm{t}(\mathrm{X} ; 18)(\mathrm{p} 11.2 ; \mathrm{q} 11.2)$ translocation that fuses SS18 (SS translocation, chromosome 18, also known as SYT) to SSX1, SSX2, or SSX4 (SS X break point 1, 2, or $4(48-51)$ ) (Figure 2a). SS18 is a ubiquitously expressed, nuclear protein that contains a novel SYT N-terminal homology domain that allows for interaction with chromatin remodeling factors and a C-terminal QPGY domain that resembles the transactivation domain within the FET family of proteins. Despite the absence of a DNA-binding domain, SS18 is thought to function as a transcriptional activator and may play a role in signal transduction via its $\mathrm{SH} 2$ (Src homology 2) and SH3 (Src homology 3)-binding motifs. The SSX genes encode a family of highly homologous proteins located on the X chromosome. They are believed to act as transcription repressors due to the presence of a Kruppel-associated box domain and an SSX repression domain. The SSX proteins also lack a DNA-binding domain so must rely on protein-protein interactions to mediate transcriptional repression (52).

The $\mathrm{t}(\mathrm{X} ; 18)$ translocation fuses the C-terminus of SSX to all but the last eight amino acids of SS18, generating a chimeric protein that contains both transcriptional activation (QPGY) and repression (SSX repression domain) domains as well as the SYT N-terminal homology domain (Figure 2a). The SSX repression domain allows SS18-SSX proteins to colocalize with components of the polycomb group chromatin remodeling repressor complex whereas the SYT N-terminal homology domain facilitates interaction with members of the switch/sucrose nonfermentable (SWI/SNF) complex $(53,54)$ (Figure 2b). This
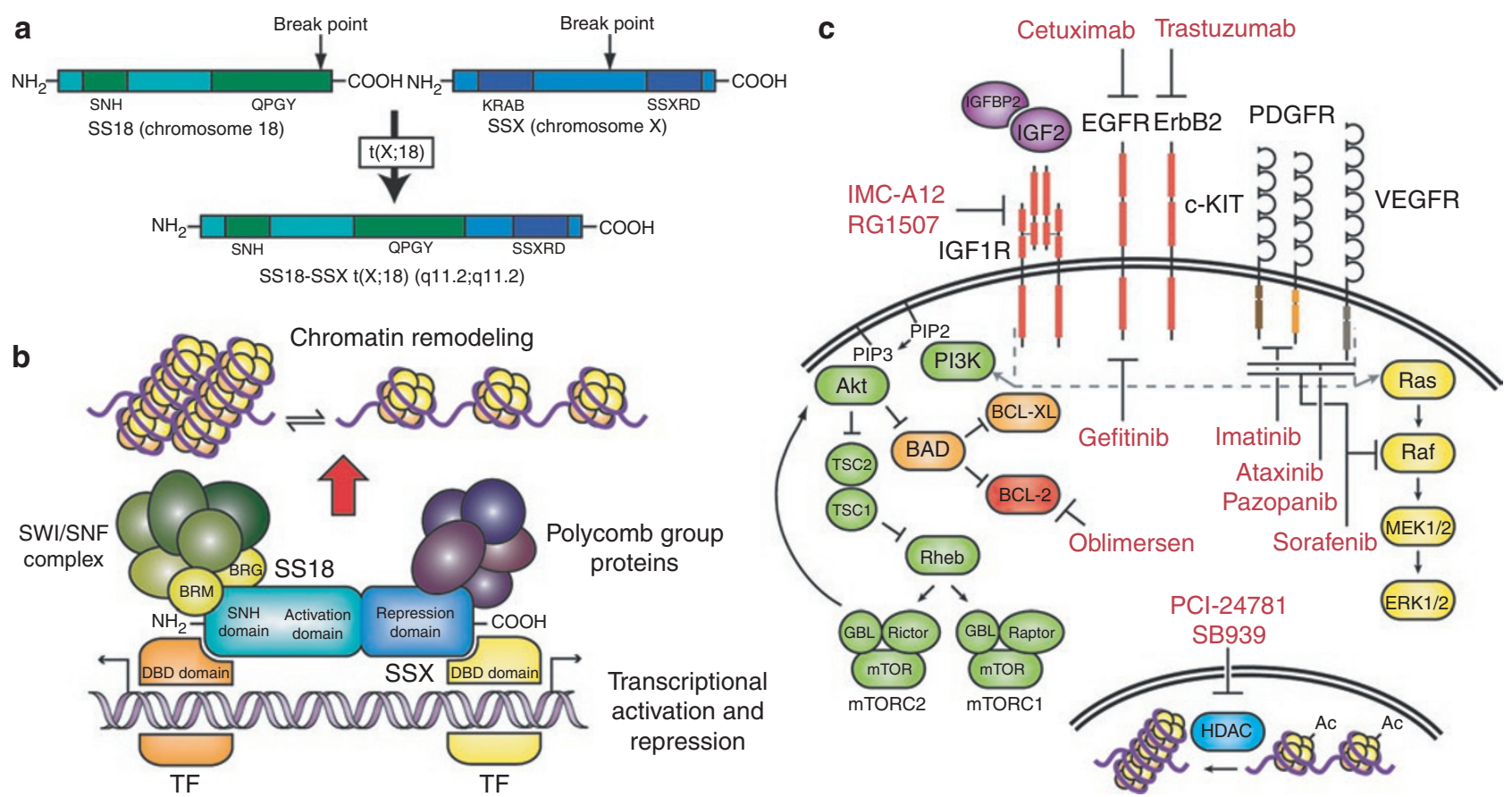

Figure 2. Molecular genetics and targeted therapies in SS. (a) Schematic of the SS18-SSX t(X;18)(q11.2;q11.2) translocation. The SS18-SSX fusion contains both the SNH and QPGY activation domains of SS18 as well as the SSXRD. The SSX KRAB domain is not retained in the fusion. (b) Putative molecular function of SS18-SSX and selected protein-protein interactions. The SNH domain facilitates binding to components of the SWI/SNF complex while the SSXRD interacts with polycomb group proteins, which results in chromatin remodeling. Interactions with transcription factors (TFs) may also lead to transcriptional activation and repression. (c) Signaling pathways and targeted therapies in SS. Activation of growth factor receptors leads to downstream signaling through the PI3K and MAPK pathways (indicated by gray dashed line and arrows). Histone deacetylases (HDACs) remove acetyl groups (Ac) from histones, resulting in condensed chromatin. Targeted therapeutic agents used in recent clinical trials for SS are indicated in red. Genes modulated by SS18-SSX are indicated in purple. Receptors overexpressed in SS are indicated in red. EGFR, epidermal growth factor receptor; IGF1, insulin-like growth factor 1; KRAB; Kruppel-associated box; MAPK, mitogen-activated protein kinase; PDGFR, platelet-derived growth factor receptor; PI3K, phosphoinositide-3-kinase; SNH, SYT N-terminal homology; SS, synovial sarcoma; SSXRD, SSX repression domain; SWI/SNF, switch/sucrose nonfermentable; SYT, synovial sarcoma translocation; VEGFR, vascular endothelial growth factor receptor. 


\section{Review $\mid$ Anderson e tal.}

suggests the fusion drives tumor progression though epigenetic chromatin remodeling. The SS18-SSX1 fusion protein is able to transform rat fibroblasts, and association with the hBRM/ hSNF2a (also known as SMARCA2, SWI/SNF-related, matrixassociated, actin-dependent regulator of chromatin, subfamily a, member 2) chromatin remodeling factor is required for transformation (55). Its presence is required for sarcomagenesis as small interfering RNA downregulation of SS18-SSX expression inhibits tumor growth in vitro and in vivo (56).

SS18 is fused to either SSX1 or SSX2 in $>90 \%$ of SS cases and is only rarely observed bound to $S S X 4$. Although initial studies showed patients with SS18-SSX2 fusions had improved survival rates, an expanded study performed more recently concluded there was no correlation between fusion variant and survival (57). Although fusion type may not determine survival, it is strongly associated with histology as biphasic and monophasic tumors contain the SS18-SSX1 and SS18-SSX2 transcripts, respectively (58).

\section{Target genes and targeted therapies}

As with RMS, IGF2 plays a role in SS biology. cDNA microarray analysis of SS and closely related spindle cell tumors revealed that genes such as those encoding IGF binding protein 2, human epidermal growth factor receptor 2 (ERBB2), and IGF2 are upregulated in SS (59). Evidence for the role of IGF2 in SS pathogenesis is further supported by studies in which SS18SSX fusions were exogenously expressed in heterologous cell systems. Gene expression profiling determined IGF2 to be the most highly upregulated gene upon SS18-SSX2 expression in $293 \mathrm{~T}$ cells (60) and SS18-SSX1 expression in human primary lung fibroblasts (61).

SS18-SSX2 has been shown to interact with Brahma-related gene 1 (BRG1) (also known as SMARCA4, SWI/SNF-related, matrix-associated, actin-dependent regulator of chromatin, subfamily a, member 4), a component of the SWI/SNF complex, and BRG1 was found to bind to the IGF2 promoter. These data combined with active chromatin marks observed upon induction of SS18-SSX2 suggest the fusion interacts with BRG1 to epigenetically modulate IGF2 expression (60). In addition, SS18-SSX2 is necessary for maintenance of IGF2 expression, and IGF2 is required for SS18-SSX1-mediated tumor formation (61). IGF2 expression results in activation of IGF1R and phosphorylation of the downstream proteins Akt (v-akt murine thymoma viral oncogene homolog) and mitogen-activated protein kinase. Providing evidence that the IGF pathway could be a therapeutic target, treatment of SS cell lines with the IGF1R inhibitor NVP-AEW541 resulted in impaired cell growth and increased apoptosis (62).

Immunohistochemical and molecular studies have demonstrated high expression levels of the antiapoptotic protein BCL-2 (B-cell CLL/lymphoma 2) in the majority of SS tumors (63). Due to the absence of genomic amplifications or rearrangements, overexpression of BCL-2 may result from transcriptional activation (64). Although BCL-2 has not been shown to be a direct target of SS18-SSX, lower mRNA and protein levels in SS tumors and cell lines lacking the $t(X, 18)$ translocation suggest an association does exist (65). Furthermore,
BCL-2 antisense oligonucleotide treatment of a translocation-positive SS cell line resulted in increased sensitivity to doxorubicin treatment, implying BCL-2 may be a promising therapeutic target (66). In a phase I trial assessing the effectiveness of BCL-2 antisense therapy (Figure 2c) in combination with chemotherapy in childhood solid tumors, two patients with SS displayed prolonged stable disease (67).

Epidermal growth factor receptor (EGFR) is also overexpressed in SS. Microarray analysis of a set of 41 soft tissue tumors and subsequent clustering analysis identified EGFR as part of cluster that showed SS-specific expression (68). Immunohistochemical studies and molecular characterization have confirmed the presence of EGFR in SS $(69,70)$. These data led to a phase II trial to establish the efficacy of the EGFR inhibitor gefitinib (Figure 2c) in EGFR-positive, chemoresistant SS. In this trial, stable disease was the best observed response, suggesting EGFR is not required for tumorigenesis (71). Gene expression profiling and immunohistochemical studies have identified another member of the EGFR family, human epidermal growth factor receptor 2 (ERBB2), that is upregulated in SS and that may provide an alternative target for the treatment of this disease $(59,70)$ (Figure 2c).

\section{DFSP}

DFSP is a relatively rare cutaneous malignancy, accounting for $\sim 0.1 \%$ of all cancers and $\sim 1 \%$ of soft tissue sarcomas. DFSP has an annual incidence rate of 4.2 per million and primarily afflicts adults between the ages of 30 and 50 years (72). Pediatric cases, both congenital and in young children, do occur, but with much less frequency than adult cases. As suggested by its name, DFSP tumors arise in the dermis, infiltrating the dermal stroma and often breaching the subcutaneous fat. Although studies have provided evidence for a fibroblastic, neural, and histiocytic origin, the histogenesis of DFSP remains uncertain (73). Primary-site locations can occur throughout the body, although the trunk, proximal extremities, and head and neck are the most common (72). The 5-year survival rate of patients with DFSP exceeds $95 \%$ and metastases occur in less than $5 \%$ of patients $(72,74)$.

Treatment of DFSP, like other soft tissue sarcomas, centers on achieving a complete surgical resection. Radiation is used when surgical margins are positive and a re-resection is not feasible. Chemotherapy is used only in metastatic cases, but there is increasing evidence that imatinib therapy can be used in an adjuvant setting in patients with recurrent, refractory, and/or metastatic disease.

\section{Molecular genetics}

DFSP tumors contain either the $t(17 ; 22)(q 22 ; q 13.1)$ reciprocal chromosomal translocation or a supernumerary ring chromosome derived from $t(17 ; 22)$. Both of these karyotypic aberrations result in a fusion of the genes COL1A1 (encoding the pro- $\alpha 1$ chain of type I collagen) on 17q21-22 and $P D G F B$ (encoding the PDGF B chain) on 22q13.1 (75) (Figure 3a). Rings are predominantly found in adult cases, although occasionally translocations are identified. In contrast, all pediatric 


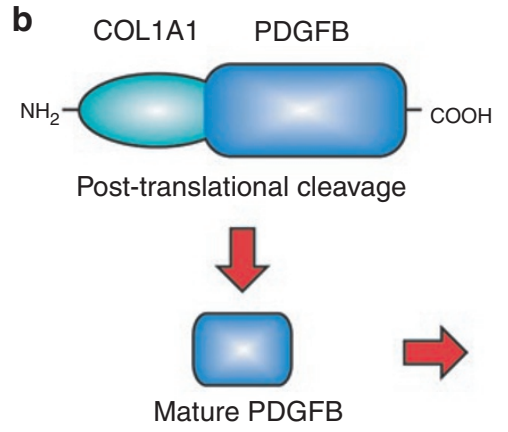

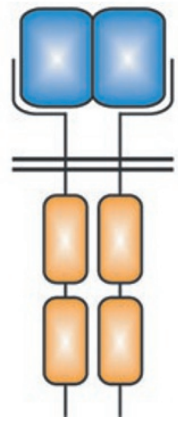

PDGFB dimer

PDGFR phosphoylation

Activation of PDGF signaling

Figure 3. Molecular genetics and targeted therapies in DFSP. (a) Schematic of the COL1A1-PDGFB t(17;22)(q22;q13.1) translocation. The COL1A1-PDGFB fusion joins the a-helical region of COL1A1 to PDGFB lacking its signal sequence. The COL1A1 N-terminal signal sequence replaces that of PDGFB. Break points occur throughout the a-helical region in COL1A1 but occur only within the first intron of PDGFB. PDGFB post-translational cleavage sites are retained in the fusion. (b) Putative molecular function of the COL1A1-PDGFB fusion. The COL1A1 signal sequence allows for protein export and posttranslational cleavage results in the generation of mature PDGFB. Ligand binding of the PDGFB homodimer dimer results in receptor dimerization, autophosphorylation, and activation. (c) Signaling pathways and targeted therapies in DFSP. Activation of PDGFR by the PDGFB dimer results in downstream signaling through the PI3K, MAPK, and Jak/Stat (Janus kinase/signal transducer and activator of transcription) pathways. VEGFR is also activated in DFSP and signals through the PI3K and MAPK pathways. Targeted therapeutic agents in current clinical trials for DFSP (imatinib and dasatinib) and soft tissue sarcomas expressing PDGFR-a (IMC-3G3) are indicated in red. Growth factors overexpressed in DFSP are indicated in red. DFSP, dermatofibrosarcoma protuberans; MAPK, mitogen-activated protein kinase; PDGFB, platelet-derived growth factor B chain; PDGFR, platelet-derived growth factor receptor; PI3K, phosphoinositide-3-kinase; VEGFR, vascular endothelial growth factor receptor.

tumors contain translocations. DFSP variants and related malignancies such as giant cell fibroblastoma have also been found to contain the COL1A1-PDGFB fusion.

The $t(17 ; 22)$ break point occurs upstream of the second exon of the PDGFB gene and within the a-helical region of COL1A1. This results in the removal of the both the PDGFB inhibitory regulatory elements and signal peptide in the COL1A1-PDGFB fusion and placement of remainder of $P D G F B$ locus, beginning with exon 2, under the control of the COL1A1 promoter. Although the $P D G F B$ break point is invariably located in the first intron, the one in the COL1A1 locus can occur within multiple exons, probably due to the repetitive nature of the $\alpha$-helical region. Most of the COL1A1 coding sequence is postulated to be functionally irrelevant as PDGFB is posttranslationally cleaved at sites retained in the fusion to generate the mature growth factor. In contrast, the replacement of the $P D G F B$ repressor elements with the COL1A1 promoter allows for aberrant expression of the protein. The COL1A1 N-terminal signal sequence, also retained in the fusion, permits PDGFB secretion, resulting in constitutive activation of the PDGF $\beta$ pathway (76) (Figure $3 \mathrm{~b}$ ). The finding that there is no correlation between COL1A1-PDGFB fusion type and clinical response or histology supports the theory that the COL1A1 portion of the fusion only provides a mechanism for PDGFB overexpression (77).

\section{Signaling pathways and targeted therapies}

The COL1A1-PDGFB fusion has been shown to transform NIH3T3 cells (78). Additionally, stable transfection of the fusion in a Chinese hamster lung fibroblastic line led to growth factor-independent growth and tumor formation in nude mice (76). Both of these studies demonstrated activation of the PDGF $\beta$ receptor, indicating the involvement of PDGF signaling in DFSP tumorigenesis. To block this pathway, NIH3T3 cells transformed with COL1A1-PDGFB and DFSP tumor-derived primary cultures were treated with imatinib mesylate (Gleevec or STI-571), which inhibits the PDGF receptor (PDGFR) (79) (Figure 3c). Growth inhibitory effects were observed in vitro and in vivo, suggesting imatinib could be an effective therapy in the treatment of DFSP.

Initial case reports demonstrating patient response to imatinib led to a study of its activity in 10 patients with locally advanced or metastatic DFSP. Despite low levels of PDGFR phosphorylation in patient tumors, imatinib was shown to be an effective therapy as four patients with locally advanced disease displayed a complete clinical response (80). On a larger scale, the efficacy of imanitib treatment is currently being evaluated in a phase II trial for DFSP and giant cell fibrosarcoma (NCT00085475, Table 2). In addition to imanitib, another tyrosine kinase inhibitor, pazopanib, which targets vascular endothelial growth 
factor receptor in addition to PDGFR (Figure 3c), has just begun phase II testing for DFSP (NCT01059656, Table 2).

\section{CONCLUSIONS}

Despite aggressive therapeutic approaches to patients with pediatric sarcomas, we have reached a plateau in the survival rate. Although the molecular genetics of these tumors reveal they are distinct entities, they are often treated as a homogeneous group when it comes to standard therapy. Fusion gene transcriptional targets, downstream signaling pathways, and overexpressed growth factor receptors provide novel therapeutic targets that are currently being investigated in clinical trials. Certain targets have shown promise, but tumor resistance is a common problem, suggesting combination therapy may be required for effective treatment. Although initial trials and in vitro studies have paved the way for advances in targeted therapy in sarcoma, further work is needed to better characterize tumors at the molecular genetic level to tailor therapies to individual tumors.

\section{STATEMENT OF FINANCIAL SUPPORT}

N.F. is supported by a St. Baldrick's Foundation Career Development Award and a STOP Cancer Award. N.F. and C.T.D. are supported by a Hyundai Hope on Wheels Grant. J.L.A. is supported by a Dissertation Year Fellowship from the Graduate Division at the University of California, Los Angeles. W.D.T. has no financial interests/support to disclose.

\section{REFERENCES}

1. Gurney JG, Swensen AR, Bulterys M. Malignant bone tumors. In: Ries LAG, Smith MA, Gurney JG, et al., eds. Cancer Incidence and Survival among Children and Adolescents: United States SEER Program 1975-1995. Bethesda, MD: National Cancer Institute, SEER Program, 1999:99-110.

2. Gurney JG, Young JL Jr, Roffers SD, Smith MA, Bunin GR. Soft tissue sarcomas. In: Ries LAG, Smith MA, Gurney JG, et al., eds. Cancer Incidence and Survival among Children and Adolescents: United States SEER Program 1975-1995. Bethesda, MD: National Cancer Institute, SEER Program, 1999:111-24.

3. Balamuth NJ, Womer RB. Ewing's sarcoma. Lancet Oncol 2010;11:184-92.

4. Rodriguez-Galindo C, Navid F, Khoury J, Krasin M. Ewing sarcoma family of tumors. In: Pappo A, ed. Pediatric Bone and Soft Tissue Sarcomas. Berlin/Heidelberg: Springer-Verlag, 2006:181-217.

5. Applebaum MA, Worch J, Matthay KK, et al. Clinical features and outcomes in patients with extraskeletal Ewing sarcoma. Cancer 2011;117:3027-32.

6. Ludwig JA. Ewing sarcoma: historical perspectives, current state-of-theart, and opportunities for targeted therapy in the future. Curr Opin Oncol 2008;20:412-8

7. Pinto A, Dickman P, Parham D. Pathobiologic markers of the ewing sarcoma family of tumors: state of the art and prediction of behaviour. Sarcoma 2011;2011:856190.

8. Barr FG, Meyer WH. Role of fusion subtype in Ewing sarcoma. J Clin Oncol 2010;28:1973-4.

9. Delattre O, Zucman J, Plougastel B, et al. Gene fusion with an ETS DNAbinding domain caused by chromosome translocation in human tumours. Nature 1992;359:162-5.

10. Kovar H. Dr. Jekyll and Mr. Hyde: The Two Faces of the FUS/EWS/TAF15 Protein Family. Sarcoma 2011;2011:837474.

11. Sharrocks AD. The ETS-domain transcription factor family. Nat Rev Mol Cell Biol 2001;2:827-37.

12. Gangwal K, Sankar S, Hollenhorst PC, et al. Microsatellites as EWS/ FLI response elements in Ewing's sarcoma. Proc Natl Acad Sci USA 2008;105:10149-54.

13. Knoop LL, Baker SJ. The splicing factor U1C represses EWS/FLI-mediated transactivation. J Biol Chem 2000;275:24865-71.

14. Knoop LL, Baker SJ. EWS/FLI alters 5'-splice site selection. J Biol Chem 2001;276:22317-22.
15. France KA, Anderson JL, Park A, Denny CT. Oncogenic fusion protein EWS/FLI1 down-regulates gene expression by both transcriptional and posttranscriptional mechanisms. J Biol Chem 2011;286:22750-7.

16. Prieur A, Tirode F, Cohen P, Delattre O. EWS/FLI-1 silencing and gene profiling of Ewing cells reveal downstream oncogenic pathways and a crucial role for repression of insulin-like growth factor binding protein 3 . Mol Cell Biol 2004;24:7275-83

17. Wakahara K, Ohno T, Kimura M, et al. EWS-Flil up-regulates expression of the Aurora A and Aurora B kinases. Mol Cancer Res 2008;6:1937-45.

18. Cironi L, Riggi N, Provero P, et al. IGF1 is a common target gene of Ewing's sarcoma fusion proteins in mesenchymal progenitor cells. PLoS ONE 2008;3:e2634.

19. Scotlandi K, Picci P. Targeting insulin-like growth factor 1 receptor in sarcomas. Curr Opin Oncol 2008;20:419-27.

20. Tolcher AW, Sarantopoulos J, Patnaik A, et al. Phase I, pharmacokinetic, and pharmacodynamic study of AMG 479, a fully human monoclonal antibody to insulin-like growth factor receptor 1. J Clin Oncol 2009;27:5800-7.

21. Pappo AS, Patel SR, Crowley J, et al. R1507, a monoclonal antibody to the insulin-like growth factor 1 receptor, in patients with recurrent or refractory Ewing sarcoma family of tumors: results of a phase II Sarcoma Alliance for Research through Collaboration study. J Clin Oncol 2011;29:4541-7.

22. Juergens H, Daw NC, Geoerger B, et al. Preliminary efficacy of the anti-insulin-like growth factor type 1 receptor antibody figitumumab in patients with refractory Ewing sarcoma. J Clin Oncol 2011;29:4534-40.

23. Malempati S, Weigel B, Ingle AM, et al. Phase I/II trial and pharmacokinetic study of cixutumumab in pediatric patients with refractory solid tumors and Ewing sarcoma: a report from the Children's Oncology Group. J Clin Oncol 2012;30:256-62.

24. Maris JM, Morton CL, Gorlick R, et al. Initial testing of the aurora kinase A inhibitor MLN8237 by the Pediatric Preclinical Testing Program (PPTP). Pediatr Blood Cancer 2010;55:26-34.

25. Toretsky JA, Erkizan V, Levenson A, et al. Oncoprotein EWS-FLI1 activity is enhanced by RNA helicase A. Cancer Res 2006;66:5574-81.

26. Erkizan HV, Kong Y, Merchant M, et al. A small molecule blocking oncogenic protein EWS-FLI1 interaction with RNA helicase A inhibits growth of Ewing's sarcoma. Nat Med 2009;15:750-6.

27. Perez EA, Kassira N, Cheung MC, Koniaris LG, Neville HL, Sola JE. Rhabdomyosarcoma in children: a SEER population based study. J Surg Res 2011;170:e243-51.

28. Pizzo PA, Poplack DG. Principles and Practice of Pediatric Oncology, 6th edn. Philadelphia: Wolters Kluwer Health/Lippincott Williams \& Wilkins, 2011:923, 980-1.

29. Barr FG, Galili N, Holick J, Biegel JA, Rovera G, Emanuel BS. Rearrangement of the PAX3 paired box gene in the paediatric solid tumour alveolar rhabdomyosarcoma. Nat Genet 1993;3:113-7.

30. Davis RJ, D'Cruz CM, Lovell MA, Biegel JA, Barr FG. Fusion of PAX7 to FKHR by the variant $\mathrm{t}(1 ; 13)$ (p36;q14) translocation in alveolar rhabdomyosarcoma. Cancer Res 1994;54:2869-72.

31. Sumegi J, Streblow R, Frayer RW, et al. Recurrent $t(2 ; 2)$ and $t(2 ; 8)$ translocations in rhabdomyosarcoma without the canonical PAX-FOXO1 fuse PAX3 to members of the nuclear receptor transcriptional coactivator family. Genes Chromosomes Cancer 2010;49:224-36.

32. Wang Q, Fang WH, Krupinski J, Kumar S, Slevin M, Kumar P. Pax genes in embryogenesis and oncogenesis. J Cell Mol Med 2008;12(6A):2281-94.

33. Fu Z, Tindall DJ. FOXOs, cancer and regulation of apoptosis. Oncogene 2008;27:2312-9.

34. Sorensen PHB, Lynch JC, Qualman SJ, et al. PAX3-FKHR and PAX7FKHR gene fusions are prognostic indicators in alveolar rhabdomyosarcoma: a report from the children's oncology group. J Clin Oncol 2002;20:2672-9.

35. Williamson D, Missiaglia E, de Reyniès A, et al. Fusion gene-negative alveolar rhabdomyosarcoma is clinically and molecularly indistinguishable from embryonal rhabdomyosarcoma. J Clin Oncol 2010;28:2151-8.

36. Ginsberg JP, Davis RJ, Bennicelli JL, Nauta LE, Barr FG. Up-regulation of MET but not neural cell adhesion molecule expression by the PAX3FKHR fusion protein in alveolar rhabdomyosarcoma. Cancer Res 1998:58:3542-6. 
37. Taulli R, Scuoppo C, Bersani F, et al. Validation of met as a therapeutic target in alveolar and embryonal rhabdomyosarcoma. Cancer Res 2006;66:4742-9.

38. Yan D, Dong Xda E, Chen X, et al. MicroRNA-1/206 targets c-Met and inhibits rhabdomyosarcoma development. J Biol Chem 2009;284:29596-604.

39. Hou J, Dong J, Sun L, et al. Inhibition of phosphorylated c-Met in rhabdomyosarcoma cell lines by a small molecule inhibitor SU11274. J Transl Med 2011;9:64

40. Ayalon D, Glaser T, Werner H. Transcriptional regulation of IGF-I receptor gene expression by the PAX3-FKHR oncoprotein. Growth Horm IGF Res 2001;11:289-97.

41. Martins AS, Olmos D, Missiaglia E, Shipley J. Targeting the insulin-like growth factor pathway in rhabdomyosarcomas: rationale and future perspectives. Sarcoma 2011;2011:209736.

42. Khan J, Bittner ML, Saal LH, et al. cDNA microarrays detect activation of a myogenic transcription program by the PAX3-FKHR fusion oncogene. Proc Natl Acad Sci USA 1999;96:13264-9.

43. Epstein JA, Song B, Lakkis M, Wang C. Tumor-specific PAX3-FKHR transcription factor, but not PAX3, activates the platelet-derived growth factor alpha receptor. Mol Cell Biol 1998;18:4118-30.

44. Taniguchi E, Nishijo K, McCleish AT, et al. PDGFR-A is a therapeutic target in alveolar rhabdomyosarcoma. Oncogene 2008;27:6550-60.

45. Abraham J, Prajapati SI, Nishijo K, et al. Evasion mechanisms to Igf1r inhibition in rhabdomyosarcoma. Mol Cancer Ther 2011;10:697-707.

46. Mayeenuddin LH, Yu Y, Kang Z, Helman LJ, Cao L. Insulin-like growth factor 1 receptor antibody induces rhabdomyosarcoma cell death via a process involving AKT and Bcl-x(L). Oncogene 2010;29:6367-77.

47. Haldar M, Hancock JD, Coffin CM, Lessnick SL, Capecchi MR. A conditional mouse model of synovial sarcoma: insights into a myogenic origin. Cancer Cell 2007;11:375-88.

48. Clark J, Rocques PJ, Crew AJ, et al. Identification of novel genes, SYT and SSX, involved in the $\mathrm{t}(\mathrm{X} ; 18)(\mathrm{p} 11.2 ; \mathrm{q} 11.2)$ translocation found in human synovial sarcoma. Nat Genet 1994;7:502-8.

49. Crew AJ, Clark J, Fisher C, et al. Fusion of SYT to two genes, SSX1 and SSX2, encoding proteins with homology to the Kruppel-associated box in human synovial sarcoma. EMBO J 1995;14:2333-40.

50. de Leeuw B, Balemans M, Olde Weghuis D, Geurts van Kessel A. Identification of two alternative fusion genes, SYT-SSX1 and SYT-SSX2, in t(X;18) (p11.2;q11.2)-positive synovial sarcomas. Hum Mol Genet 1995;4:1097-9.

51. Skytting B, Nilsson G, Brodin B, et al. A novel fusion gene, SYT-SSX4, in synovial sarcoma. J Natl Cancer Inst 1999;91:974-5.

52. Haldar M, Randall RL, Capecchi MR. Synovial sarcoma: from genetics to genetic-based animal modeling. Clin Orthop Relat Res 2008;466:2156-67.

53. Perani M, Ingram CJE, Cooper CS, Garrett MD, Goodwin GH. Conserved SNH domain of the proto-oncoprotein SYT interacts with components of the human chromatin remodelling complexes, while the QPGY repeat domain forms homo-oligomers. Oncogene 2003;22:8156-67.

54. Soulez M, Saurin AJ, Freemont PS, Knight JC. SSX and the synovial-sarcoma-specific chimaeric protein SYT-SSX co-localize with the human Polycomb group complex. Oncogene 1999;18:2739-46.

55. Nagai M, Tanaka S, Tsuda M, et al. Analysis of transforming activity of human synovial sarcoma-associated chimeric protein SYT-SSX1 bound to chromatin remodeling factor hBRM/hSNF2 alpha. Proc Natl Acad Sci USA 2001;98:3843-8.

56. Takenaka S, Naka N, Araki N, et al. Downregulation of SS18-SSX1 expression in synovial sarcoma by small interfering RNA enhances the focal adhesion pathway and inhibits anchorage-independent growth in vitro and tumor growth in vivo. Int J Oncol 2010;36:823-31.

57. Guillou L, Benhattar J, Bonichon F, et al. Histologic grade, but not SYTSSX fusion type, is an important prognostic factor in patients with synovial sarcoma: a multicenter, retrospective analysis. J Clin Oncol 2004;22:4040-50.

58. Antonescu CR, Kawai A, Leung DH, et al. Strong association of SYT-SSX fusion type and morphologic epithelial differentiation in synovial sarcoma. Diagn Mol Pathol 2000;9:1-8.

59. Allander SV, Illei PB, Chen Y, et al. Expression profiling of synovial sarcoma by cDNA microarrays: association of ERBB2, IGFBP2, and ELF3 with epithelial differentiation. Am J Pathol 2002;161:1587-95.
60. de Bruijn DR, Allander SV, van Dijk AH, et al. The synovial-sarcomaassociated SS18-SSX2 fusion protein induces epigenetic gene (de)regulation. Cancer Res 2006;66:9474-82.

61. Sun Y, Gao D, Liu Y, Huang J, Lessnick S, Tanaka S. IGF2 is critical for tumorigenesis by synovial sarcoma oncoprotein SYT-SSX1. Oncogene 2006;25:1042-52.

62. Friedrichs N, Küchler J, Endl E, et al. Insulin-like growth factor-1 receptor acts as a growth regulator in synovial sarcoma. J Pathol 2008;216:428-39.

63. Krsková L, Kalinová M, Brízová $H$, Mrhalová $M$, Sumerauer D, Kodet R. Molecular and immunohistochemical analyses of BCL2, KI-67, and cyclin D1 expression in synovial sarcoma. Cancer Genet Cytogenet 2009;193:1-8.

64. Mancuso T, Mezzelani A, Riva C, et al. Analysis of SYT-SSX fusion transcripts and bcl-2 expression and phosphorylation status in synovial sarcoma. Lab Invest 2000;80:805-13.

65. Albritton KH, Randall RL. Prospects for targeted therapy of synovial sarcoma. J Pediatr Hematol Oncol 2005;27:219-22.

66. Joyner DE, Albritton KH, Bastar JD, Randall RL. G3139 antisense oligonucleotide directed against antiapoptotic Bcl-2 enhances doxorubicin cytotoxicity in the FU-SY-1 synovial sarcoma cell line. J Orthop Res 2006;24:474-80.

67. Rheingold SR, Hogarty MD, Blaney SM, et al.; Children's Oncology Group Study. Phase I Trial of G3139, a bcl-2 antisense oligonucleotide, combined with doxorubicin and cyclophosphamide in children with relapsed solid tumors: a Children's Oncology Group Study. J Clin Oncol 2007;25:1512-8.

68. Nielsen TO, West RB, Linn SC, et al. Molecular characterisation of soft tissue tumours: a gene expression study. Lancet 2002;359:1301-7.

69. Bozzi F, Ferrari A, Negri T, et al. Molecular characterization of synovial sarcoma in children and adolescents: evidence of akt activation. Transl Oncol 2008;1:95-101.

70. Thomas DG, Giordano TJ, Sanders D, et al. Expression of receptor tyrosine kinases epidermal growth factor receptor and HER-2/neu in synovial sarcoma. Cancer 2005;103:830-8.

71. Ray-Coquard I, Le Cesne A, Whelan JS, et al. A phase II study of gefitinib for patients with advanced HER-1 expressing synovial sarcoma refractory to doxorubicin-containing regimens. Oncologist 2008;13:467-73.

72. Criscione VD, Weinstock MA. Descriptive epidemiology of dermatofibrosarcoma protuberans in the United States, 1973 to 2002. J Am Acad Dermatol 2007;56:968-73.

73. Dominguez-Malagon H, Valdez-Carrillo Mdel C, Cano-Valdez AM. Dermatofibroma and dermatofibrosarcoma protuberans: a comparative ultrastructural study. Ultrastruct Pathol 2006;30:283-91.

74. Iqbal CW, St Peter S, Ishitani MB. Pediatric dermatofibrosarcoma protuberans: multi-institutional outcomes. J Surg Res 2011;170:69-72.

75. Simon MP, Pedeutour F, Sirvent N, et al. Deregulation of the platelet-derived growth factor B-chain gene via fusion with collagen gene COL1A1 in dermatofibrosarcoma protuberans and giant-cell fibroblastoma. Nat Genet 1997; 15:95-8.

76. Simon MP, Navarro M, Roux D, Pouysségur J. Structural and functional analysis of a chimeric protein COL1A1-PDGFB generated by the translocation $\mathrm{t}(17 ; 22)(\mathrm{q} 22 ; \mathrm{q} 13.1)$ in Dermatofibrosarcoma protuberans (DP). Oncogene 2001;20:2965-75.

77. Giacchero D, Maire G, Nuin PA, et al. No correlation between the molecular subtype of COL1A1-PDGFB fusion gene and the clinico-histopathological features of dermatofibrosarcoma protuberans. J Invest Dermatol 2010;130:904-7.

78. Greco A, Fusetti L, Villa R, et al. Transforming activity of the chimeric sequence formed by the fusion of collagen gene COL1A1 and the platelet derived growth factor b-chain gene in dermatofibrosarcoma protuberans. Oncogene 1998;17:1313-9.

79. Greco A, Roccato E, Miranda C, Cleris L, Formelli F, Pierotti MA. Growthinhibitory effect of STI571 on cells transformed by the COL1A1/PDGFB rearrangement. Int J Cancer 2001;92:354-60.

80. McArthur GA, Demetri GD, van Oosterom A, et al. Molecular and clinical analysis of locally advanced dermatofibrosarcoma protuberans treated with imatinib: Imatinib Target Exploration Consortium Study B2225. J Clin Oncol 2005;23:866-73. 